\title{
Disentangling the Role of Religiosity in Human Papillomavirus Vaccination Amidst COVID-19 Pandemic
}

\author{
Ayokunle A. Olagoke ${ }^{1}$ (1) $\cdot$ Brenikki Floyd ${ }^{2} \cdot$ Rachel Caskey $^{2,3}$. \\ Jennifer Hebert-Beirne ${ }^{2}$. Andrew D. Boyd ${ }^{4}$. Yamile Molina ${ }^{2,5}$
}

Accepted: 20 December 2021 / Published online: 3 February 2022

(c) The Author(s), under exclusive licence to Springer Science+Business Media, LLC, part of Springer Nature 2022

\begin{abstract}
Religion is a complex and sociocultural driver of human papillomavirus (HPV) vaccination decisions, but its exact role has been mixed/unclear. We used a crosssectional study of 342 Christian parents to examine the associations between the three domains of religiosity (organizational, non-organizational, and intrinsic) and the intention to (i) seek HPV information and (ii) receive the HPV vaccine. Organizational religiosity was the only domain that was positively associated with information-seeking intention regardless of the type of covariates included. Mixed findings in the association between religiosity and HPV vaccination decisions may depend on the religiosity domain being assessed.
\end{abstract}

Keywords Religiosity $\cdot$ Spirituality $\cdot$ Human papillomavirus (HPV) $\cdot$ HPV vaccination $\cdot$ COVID-19 $\cdot$ Intention $\cdot$ Muslims $\cdot$ Christians

Ayokunle A. Olagoke

aolagoke@wustl.edu

1 Health Communication Research Laboratory, The Brown School, Washington University in St. Louis, St. Louis, MO 63130, USA

2 Division of Community Health Sciences, School of Public Health, University of Illinois at Chicago, Chicago, IL, USA

3 Department of Medicine, University of Illinois at Chicago, Chicago, IL, USA

4 Department of Biomedical and Health Information Science, University of Illinois at Chicago, Chicago, IL, USA

5 Center for Research on Women and Gender, College of Medicine, University of Illinois, Chicago, IL, USA 


\section{Introduction}

Human papillomavirus (HPV) is the most common sexually transmitted disease in the USA (CDC, 2019), with an annual financial cost estimated at 7 billion dollars (Perkins et al., 2015). The HPV vaccine has been available since 2006 and is universally recommended at 11-12 years of age for both boys and girls (Meites et al., 2016). Vaccinated adolescents are more likely to have long-lasting protection against HPV-related infections and cancers than unvaccinated adolescents (Brotherton, 2019; Näsman et al., 2020). Despite the substantial benefits of HPV vaccination, nearly half of eligible adolescents have not completed the HPV vaccine series (CDC, 2021). Though distinct, the intentions to vaccinate and to seek HPV information are considered important intermediate antecedents of actual HPV vaccination behavior (Catalano et al., 2017; Hamilton et al., 2020). This current study investigates factors influencing these intentions.

\section{Religiosity as a Crucial, Complex Predictor of HPV Vaccination Behaviors}

A commonly studied primary predictor of HPV vaccination behaviors is religiosity. In this study, we operationalized religiosity as participating in group or private activities that reflect one's belief in the existence of God or a higher power (Okunrounmu et al., 2016). Christianity is the most well-studied religious group (Best et al., 2019; Birmingham et al., 2019; Marti et al., 2017), likely because it is the most common religions. Some studies have shown that HPV vaccination intention and uptake are suboptimal among highly religious groups (Best et al., 2019; Marti et al., 2017). Commonly cited reasons for vaccine decline among these religious populations include fears that the HPV vaccine encourages a child's promiscuity and the belief that supernatural powers control health outcomes (Best et al., 2019; Padela et al., 2018; Pratt et al., 2019). On the other hand, other studies have shown positive or nonsignificant associations between religiosity and HPV vaccination intention (Brueggmann et al., 2016; Mupandawana \& Cross, 2016; Thomas et al., 2015), making it challenging to clarify the exact role of religiosity in the vaccination against HPV.

These conflicting findings may partly be due to the narrow measurement of religiosity, a broad, multi-domain construct. Specifically, existing findings have not considered which religiosity domains are most strongly associated with the HPV vaccination and information-seeking intentions. Therefore, previously mixed findings on the association between religiosity and HPV vaccination may have been due to the use of inconsistent or insensitive religiosity measurement. This inconsistency involves (i) assessing one domain of religiosity at a time (e.g., religious affiliation or service attendance (Brueggmann et al., 2016; Wong et al., 2019) without accounting for the influence of other domains or (ii) not distinguishing between domains (Best et al., 2019; Birmingham et al., 2019). In this current study, we deconstructed religiosity into three domains using Koenig 
(2010)'s classification (as stated below) and examined their associations with HPV vaccination decisions (Koenig \& Büssing, 2010):

(1) organizational religious activities (ORA), defined as participating in public or group-related religious activities like attending religious services, group prayers, or fellowship;

(2) non-organizational religious activities (NORA), defined as self-directed private religious activities or devotion such as personal prayers and scripture readings, watching religious programs online or on the television, and other personalized rituals; and

(3) intrinsic religious activities (IRA), defined as considering major life decisions and perceptions of the world (e.g., unjustified hope for relief, greater life meaning, purpose in life, self-control) in the context of one's personalized religious views.

Practicing ORA may be positively associated with HPV information-seeking intention through factors such as trust in local faith leaders as credible sources of health information (Birmingham et al., 2019; Bodson et al., 2017). Therefore, religious leaders may leverage their influence to advocate for HPV vaccination as a viable strategy for preventing HPV-related infections. Also, previous studies suggest that participation in ORA through regular service attendance may expose a parent to the activities of faith-based health ministries and outreaches that offer venue-based health education programs (Brandt et al., 2019; Lahijani et al., 2021). We hypothesized that ORA would be associated with a higher intention to seek HPV information and to vaccinate a child.

Practicing NORA (marked by private and selective religious rituals) may exclude a person from group/venue-based religious events where there is a deliberate effort to leverage religiosity to influence HPV vaccination decisions. This exclusion may lead a person who practices NORA to have personal beliefs about HPV vaccination that are different from their denomination (Mupandawana \& Cross, 2016). Hence, unlike ORA, where the religious organization may structure events to influence members' vaccination decisions, practicing NORA may limit parents' exposure to religious programs that promote HPV vaccination awareness. We, therefore, hypothesized that NORA would be unassociated with HPV information-seeking and vaccination intentions.

Similarly, IRA, marked by beliefs such as perceived closeness to God and filtering life decisions (such as vaccination) through the lens of one's religious beliefs, may be negatively associated with HPV vaccination intention (Bodson et al., 2017). Past studies have established that dimensions of religiosity such as spirituality may negatively influence vaccination decisions (da Silva Oliveira et al., 2017). A parent who practices IRA may filter their HPV vaccination decision through the external locus of control beliefs and consider it unnecessary to seek HPV information or vaccination (Olagoke et al., 2020). We, therefore, hypothesized that IRA would be negatively associated with HPV informationseeking and vaccination intentions. 


\section{Current Study}

This current study sheds more light on the previously mixed findings regarding religiosity and HPV vaccination. Specifically, past studies have examined religiosity as a monolithic construct in its relationship with HPV vaccination. They have not consistently operationalized religiosity in terms of the three identified domains (ORA, NORA, and IRA) in our study, which appear to differ in their independent associations with HPV vaccination decisions. Our study offered a critical investigation into the above-mentioned gap identified among Christians parents of unvaccinated adolescents. We examined the associations between the three domains of religiosity and information-seeking and vaccination intentions.

\section{Methods}

We used a cross-sectional online survey from September 28 to October 8, 2020. The Institution Review Board of the University of Illinois Chicago approved all study materials and procedures.

\section{Recruitment Procedures and Sample}

Participants were recruited through the Qualtrics Panel System (QPS). The QPS is an online survey research platform that offers access to a large and diverse group of individuals across North America interested in contributing to research. It has become a popular and reliable recruitment method for researchers interested in conducting observational (cross-sectional and longitudinal) and experimental research (Bryan et al., 2020; Hamm \& Yun, 2019; Soucy \& Hadjistavropoulos, 2017).

QPS recruits respondents through multiple methods such as member referrals, targeted email lists, permission-based networks, and social media. To register with QPS, participants are required to complete background questionnaires and agree to partake in online surveys for an incentive/reward. QPS determines the minimum incentive by considering factors like the incidence rate of the condition studied and the survey's length. Incentives may include cash, airline miles, and gift cards. The QPS uses the profile information provided by registered respondents to randomly select potential participants who are likely to meet the study eligibility criteria. Potential participants are sent an invitation link via their email with the following information: (1) clarification that the survey is for research purposes only; (2) expected survey length; and (3) incentive for participation. Specific details about the study are not included to avoid selection biases.

For this study, we used a two-step screening procedure to determine eligibility. First, QPS invited participants via email. Screening criteria included (i) being over 18 years of age at the time of recruitment, (ii) being parents or guardians of at least one child aged 11-17 years who have not received any dose of the HPV 
vaccine, (iii) identifying as a Christian, and (iv) residing in the USA. QPS also enforced the eligibility screening criteria by activating the fraud detection settings to detect fraudulence in the use of IP addresses.

As a second screening measure, we further ensured data quality by excluding participants who completed the survey less than half the median completion time $(8 \mathrm{~min}$ ) who may have been inattentive or sped through the survey. We also subtracted the child's reported age from the parents' as a response validity check. Participants whose parent-child age difference showed that they were 8-14 years old at the time of birth were removed from the study. Finally, we included instructional manipulation checks in various survey sections to improve attentiveness during survey completion. At the beginning of the survey, respondents were informed that their attention would be checked and that inattentive participants would be discontinued from proceeding (Paas \& Morren, 2018). We asked participants to skip specific questions to check if they were attentive to the instructions. Participants who failed the attention checks were automatically screened out of the survey.

In total, we assessed 969 potential participants for eligibility. Of them, 502 met the eligibility criteria and yielded a final analytic sample of 342 participants (Fig. 1).

\section{Data Collection Procedures and Measures}

Eligible participants were directed to an online informed consent form, which they completed before proceeding with the survey. Eligible participants completed surveys between 7.96 and $109.87 \mathrm{~min}$ (median completion time $15.92 \mathrm{~min}$ ). When answering questions regarding their child/children, if participants have more than one child between 11 and 17 years old, they were asked to think of the oldest eligible child.

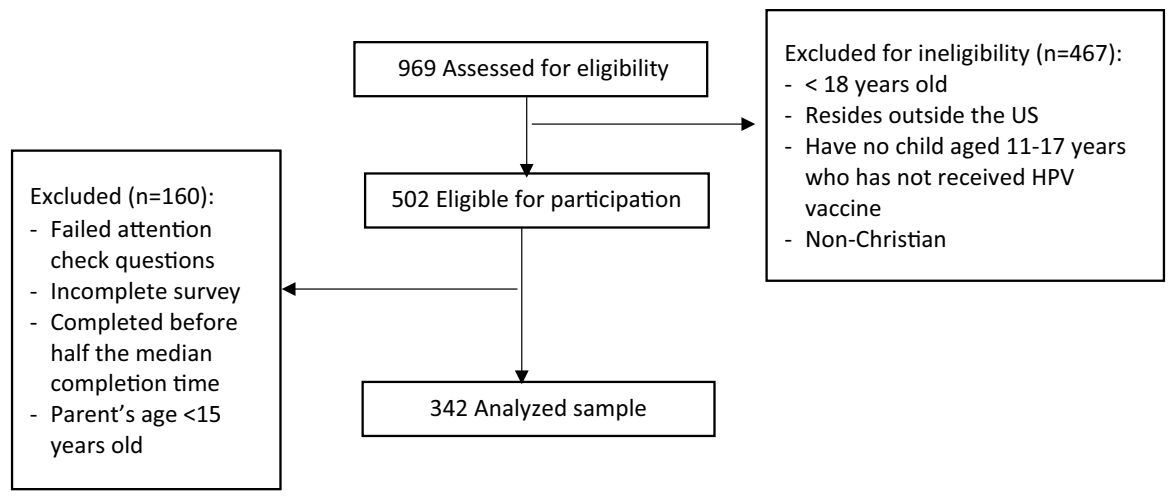

Fig. 1 Sample and recruitment 


\section{Dependent Variables}

Intention to seek HPV information was assessed with a two-item measure $(\alpha=0.92$; Abhyankar et al., 2008) on a 5-point Likert scale of "Definitely false" to "Definitely true." Parents were asked about their (i) intention and (ii) wish to seek health information regarding HPV in the next two weeks. The mean of the responses was analyzed, thereby ranging from 1 to 5.

$H P V$ Vaccination intention was assessed with a two-item measure $(\alpha=0.90$; Abhyankar et al., 2008) on a 5-point Likert scale of "Definitely false" to "Definitely true." Participants were asked if they (i) intended and (ii) wished to vaccinate their children against HPV in the next 1 year. The mean of the responses was analyzed, thereby ranging from 1 to 5 .

\section{Independent Variables}

Religiosity domains. We measured the three religiosity domains of interest by adapting the 5-item Duke University Religion Index (DUREL), which has been used in over 100 studies (Koenig \& Büssing, 2010). The DUREL scale has shown a high 2 -week test-retest reliability of 0.91 , a reliable internal consistency $(\alpha=0.78-0.91)$, and convergent validity with other established measures of religiosity $(r$ 's $=0.71-0.86)$.

Organized religious activity (ORA) is the first subscale and first question in the original DUREL that asked about the frequency of attendance at religious services. Because of the current COVID-19 pandemic, we modified this subscale by asking two separate questions to assess the domain. One item referred to in-person or online attendance between 2015 and January 2020, while the second item assessed in-person or online attendance since January 2020. The Cronbach's alpha for these items was 0.64 . The mean score (ranged from 1 to 5.5) was used in the analysis.

Non-organized religious activity (NORA) — the second subscale, was measured using the second question in the original DUREL. This item measured the frequency of private religious activities such as prayer and scripture reading. Responses ranged from Never (1) to Daily (5).

Intrinsic religious activity (IRA) is the third subscale $(\alpha=0.74)$. It was measured using the remaining three statements in the original DUREL. For example, "My religious beliefs are what really lie behind my whole approach to life." Options ranged from Definitely true (1) to Definitely false (5). Responses were reversely coded such that lower numbers indicated less certainty.

\section{Sociodemographic, Socioeconomic, and Health-Related Covariates}

Participants reported on the following important sociodemographic characteristicsparent's age (continuous variable), age of the child, parent's sex (female, male), sex of the (eldest eligible) child; race/ethnicity that was based on distinct self-reported race and Hispanic ethnicity; and marital status (married, divorced, separated, 
widowed, or single). Socioeconomic status (SES) characteristics included household income $(<\$ 20,000, \$ 20,000-<\$ 35,000, \$ 35,000-<\$ 50,000, \$ 50,000-<\$ 75,000$, and $\$ 75,000$ or more); employment status, and education (less than high school, high school graduate, some college, college graduate or more). Health-related factors were measured using two items that measured HPV-related family medical history and general vaccine beliefs (Abhyankar et al., 2008). Specifically, participants were asked: (1) if they or their family members have been diagnosed with HPVrelated infections like cervical cancer, penile cancer, genital warts, anal cancer (Yes or No), and (2) if they had a personal belief against vaccines in general (Yes or No).

\section{Analytical Strategy}

To assess the distribution of continuous variables, we examined skewness and kurtosis. Values between -2 and 2 were considered to be normally distributed (White, 2013). Descriptive statistics such as mode (to ascertain bimodal distributions), mean, standard deviations, and frequencies were also calculated for Likert scales. Next, we conducted bivariate linear regression models to examine associations between the independent variables (i.e., the three domains of religiosity) and dependent variables (i.e., intention to seek HPV information and intention to vaccinate). Finally, we conducted separate multiple linear regressions for each dependent variable. We included all three domains into all models to test the independent association of each domain with our dependent variable.

We had a two-step process to address the effects of potential covariates. First, we had partial models. We examined models that included all three independent variables and one covariate domain, as conceptualized by Andersen and Newman's model (Andersen \& Newman, 1973; Molina et al., 2015). Second, we had full models. Here, we included all three independent variables and covariates into our models. $p$ values $<0.05$ were considered to be significant in all models.

\section{Results}

Table 1 depicts the participants' characteristics. Respondents had a mean age of $41.3 \pm 5.5$ years and a child's age of $14.1 \pm 2.1$ years. Approximately half of the sample was male $(54.1 \%)$. Respondents mostly had male children $(65.7 \%)$, were White $(87.1 \%)$, married $(88.9 \%)$, had a college education or more $(74.9 \%)$, and were employed $(83.3 \%)$. There were $228(64.6 \%)$ participants who had no personal belief against vaccines and $335(92.7 \%)$ without a history of HPV-related infections. The mean values for the religiosity domains were $3.1 \pm 0.9$ (for ORA), $3.5 \pm 1.4$ (for NORA), and $4.3 \pm 0.8$ (for IRA). The mean intention to vaccinate a child against HPV in the next year was $3.1 \pm 1.4$, while the mean intention to seek HPV information was $3.1 \pm 1.3$. Kurtosis and skewness of all continuous variables ranged from -0.21 to 1.74 , indicating that they were normally distributed.

Table 2 describes the associations between the domains of religiosity and information-seeking intention after controlling for the three categories of covariates. 
Table 1 Participants' characteristics $(N=342)$
Participants' characteristics
Sociodemographic characteristics

Parent's age

Child's age

Parent's sex

Female

Male

Child's sex

Female

Male

Race

White

Non-White

Marital status

Not married

Married

Denomination

Catholic

Non-Catholic

Socioeconomic status characteristics

Highest education

Less than a college degree

$86(25.1 \%)$

College or more

Household income

Less than $\$ 75,000$

Over $\$ 75,000$

Employment status

Employed

Unemployed/retired/disabled/others

Health-related factors

Personal belief against vaccines in general

Yes/not sure

No

$121(35.4 \%)$

$221(64.6 \%)$

History of HPV-related infections

Yes

No

$317(92.7 \%)$

Religiosity domains

Organizational religiosity

Non-organizational religiosity

Intrinsic religiosity

$4.3(0.8)$

Outcome variables

Intention to seek HPV information

$3.1(1.4)$

Intention to vaccinate against HPV
$3.1(1.3)$ 


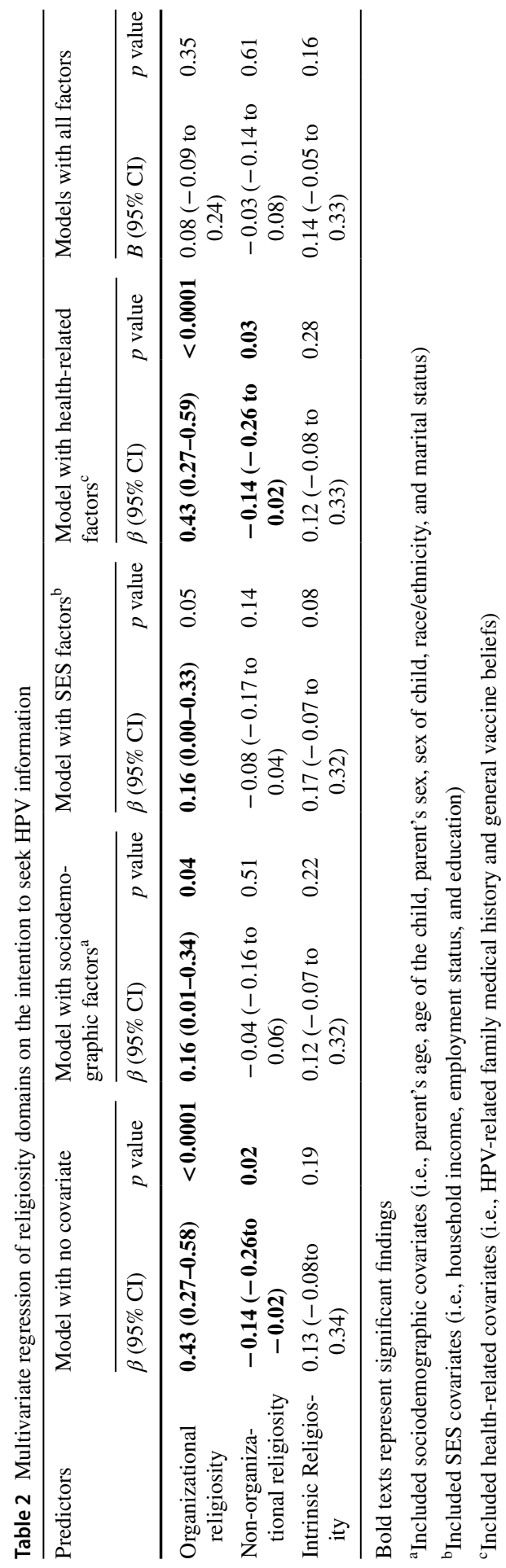


ORA was positively associated with intention to seek HPV information in the models that included (i) sociodemographic factors $(\beta=0.16 ; 0.01-0.34 ; p=0.04)$, (ii) SES factors $(\beta=0.16 ; 0.00-0.33 ; p=0.05)$, and (iii) health-related factors $(\beta=0.43 ; 0.27-0.59 ; p=<0.0001)$. NORA was negatively associated with the intention to seek HPV information in the model that included health-related covariates $(\beta=-0.14 ;-0.26$ to $-0.02 ; p=0.03)$. Associations between IRA and informationseeking intention were not significant across different models. To note, none of the different religiosity domains were significantly associated with the intention to seek HPV information in the full model. Similarly, as shown in Table 3, none of the three religiosity domains were associated with the intention to vaccinate in both the partially and fully adjusted models.

\section{Discussion}

This study is the first to compare the associations between the three domains of religiosity and HPV information-seeking and vaccination intentions. Overall, our findings showcase how the association between religiosity and HPV vaccination decisions (i.e., information-seeking and vaccination intentions) depends on which domain is being studied and what covariates are accounted for. Specifically, the ORA domain appears to have a stronger and more consistent positive association (than other domains) with the intention to seek HPV information regardless of the types of covariates included. However, none of the religiosity domains was associated with HPV vaccination intention.

Our findings support previous studies highlighting the role of religious centers in hosting faith-based health ministries, community engagement/mobilization events, and social change communication outreaches to create awareness about HPV vaccination to their members (Brandt et al., 2019; Lahijani et al., 2021). These events may have exposed parents who practice ORA to HPV-related information, causing them to report higher intention to seek HPV information. However, informationseeking may not automatically lead to vaccination intention. There is evidence of mixed messaging regarding HPV vaccination among religious people (Manika et al., 2017; Touyz \& Touyz, 2013). Information-seeking may only lead to vaccination intention if the message that is being sought addresses the existing barrier beliefs that religious parents have regarding vaccination (Bodson et al., 2017; Gordon et al., 2011; Kreuter et al., 2012; Mupandawana \& Cross, 2016; Weinstein et al., 2016). Therefore, it is crucial to empower religious centers to provide accurate HPV vaccination information that addresses parents' concerns.

The nonsignificant association between the three domains of religiosity and intention to vaccinate in all the models is similar to past research findings (Birmingham et al., 2019; Brueggmann et al., 2016; Shelton et al., 2013). This suggests that, first, a person may not have an intention to vaccinate their child simply because they attend religious services (ORA), participate in private fellowship (NORA), or personally interpret scriptures (IRA). Instead, religiosity may only influence HPV vaccination if the religious organizations and stakeholders deliberately plan and structure their religious activities to impact their vaccination intention. Our findings 


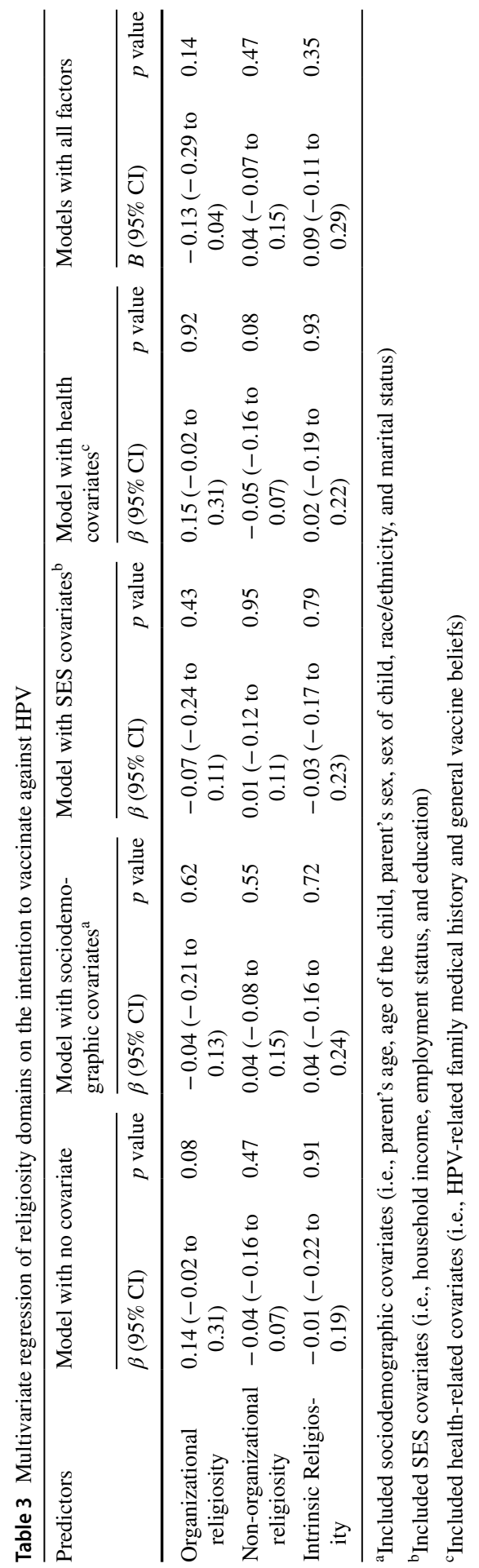


further indicate a need to design faith-based messaging interventions that address religious barrier beliefs to HPV vaccination among parents who practice these dimensions of religiosity.

Second, past findings have shown that it may be challenging for faith leaders to advocate for HPV vaccination for adolescents because it is considered controversial. Religious leaders have expressed discomfort and having to tread carefully on discussions around the sexual activity of adolescents (Lahijani et al., 2021; Touyz \& Touyz, 2013; Williams et al., 2020). They have regarded it as a complicated and sensitive topic to discuss in their capacity as religious leaders (either through their services or mass media programs). Therefore, it is essential to engage with faith leaders and determine how best they can be supported and empowered to promote HPV vaccination if they would like to (Lahijani et al., 2021). This inquiry may be useful in increasing HPV vaccination through religious settings.

Third, this study was conducted at the peak of a global pandemic where there were restrictions to movement, uptake of preventive services (such as HPV vaccination), and outpatient visits. Hence, parents may be uncertain about the possibility of vaccinating their children in the next year (as measured in this study). Recent evidence suggests that in the first half of 2020, HPV vaccination and other cancer screening services have been disrupted in many developed countries due to the pandemic (Arbyn et al., 2020). Therefore, due to this uncertainty, parents may intend to seek information about HPV and not intend to vaccinate their adolescents in the next year until some form of normalcy is restored to HPV vaccination activities and hospital visits are considered.

\section{Study Limitations}

Our study is not without its limitations. First, although previous studies have identified information-seeking intention as a proximal predictor of HPV vaccination (Lee \& Kim, 2015), we did not validate this because our study was underpowered (a potential source of type II error) to explore a mediating effect. Therefore, future studies should test the mediating role of information-seeking intention. This will further confirm if information-seeking is the primary driver of the effect of religiosity on vaccination intention and can guide intervention design.

Second, there are other dimensions of religiosity that our measure may not have captured. Future studies should examine the role of religiosity in HPV vaccination by exploring other domains that may have been excluded in our study. Third, our use of online and convenient sampling threatens our studies' generalizability across nonInternet users, time (e.g., a period of a global pandemic), religious denominations, and all parents of adolescents. Nevertheless, the robust quality of our data provides an opportunity to make a significant contribution to our subject matter. Also, we only assessed parents of unvaccinated children, making it difficult to test the extent to which HPV vaccination status varies by religiosity. More research is needed to explore if parents differ in their HPV vaccination decision based on their religiosity. Finally, religiosity may have changed in many ways beyond comprehension due to the pandemic, thereby impacting its association with our outcome. 


\section{Conclusion and Implications}

In conclusion, organizational religiosity, through frequent religious service attendance (in-person and online), may serve as the most vital dimension of religiosity to be positively associated with HPV vaccination among religious parents. Future studies are needed to identify the specific activities in religious settings that increase HPV information-seeking intention. Factors such as trust in local faith leaders, personal beliefs, and faith-based ministry that outreaches through community engagements/mobilizations should be explored (Lahijani et al., 2021).

Furthermore, since information-seeking does not always lead to vaccination intention, it is important to move parents from information-seeking to actual vaccination behavior. This can be facilitated by conducting in-depth qualitative research to understand the type of HPV information parents are exposed to, their interpretation of the message, and the barriers they face in vaccinating their children after exposure to accurate HPV information.

Authors' Contributions All authors contributed to the study conception and design. Material preparation, data collection, and analysis were performed by Ayokunle Olagoke and Yamile Molina. The first draft of the manuscript was written by Ayokunle Olagoke, and all authors commented on previous versions of the manuscript. All authors read and approved the final manuscript.

Data Availability The data that support the findings of this study are not publicly available due to privacy or ethical restrictions.

\section{Declarations}

Conflict of Interest The authors declare that they have no conflict of interest.

Consent to Participate Informed consent was obtained from all individual participants included in the study.

Consent to Publish The participants consented to the dissemination of the study findings through scholarly publications.

Ethics Approval Approval was obtained from the ethics committee of the University of Illinois at Chicago. The procedures used in this study adhere to the tenets of the Declaration of Helsinki.

Human Participants and/or Animals This article does not contain any studies with animals performed by any of the authors. Ethical approval was obtained from the Institution review board of the University of Illinois at Chicago. The procedures used in this study, involving human participants, adhered to the tenets of the 1964 Declaration of Helsinki and its later amendments or comparable ethical standards.

Informed Consent Standard procedures for obtaining consent and maintaining the confidentiality and anonymity of participants were instituted. Informed consent was obtained from all individual participants included in the study. 


\section{References}

Abhyankar, P., O'connor, D. B., \& Lawton, R. (2008). The role of message framing in promoting MMR vaccination: Evidence of a loss-frame advantage. Psychology, Health and Medicine, 13(1), 1-16. https://doi.org/10.1080/13548500701235732

Andersen, R., \& Newman, J. F. (1973). Societal and individual determinants of medical care utilization in the United States. The Milbank Memorial Fund Quarterly. Health and Society, 51(1), 95-124. https://doi.org/10.2307/3349613

Arbyn, M., Bruni, L., Kelly, D., Basu, P., Poljak, M., Gultekin, M., Bergeron, C., Ritchie, D., \& Weiderpass, E. (2020). Tackling cervical cancer in Europe amidst the COVID-19 pandemic. The Lancet. Public Health, 5(8), e425. https://doi.org/10.1016/S2468-2667(20)30122-5

Best, A. L., Thompson, E. L., Adamu, A. M., Logan, R., Delva, J., Thomas, M., Cunningham, E., Vamos, C., \& Daley, E. (2019). Examining the influence of religious and spiritual beliefs on HPV vaccine uptake among college women. Journal of Religion and Health, 58(6), 2196-2207. https://doi.org/ 10.1007/s10943-019-00890-y

Birmingham, W. C., Macintosh, J., Vaughn, A. A., \& Graff, T. C. (2019). Strength of belief: Religious commitment, knowledge, and HPV vaccination adherence. Psycho-Oncology, 28(6), 1227-1233. https://doi.org/10.1002/pon.5071

Bodson, J., Wilson, A., Warner, E. L., \& Kepka, D. (2017). Religion and HPV vaccine-related awareness, knowledge, and receipt among insured women aged 18-26 in Utah. PLoS ONE, 12(8), e0183725. https://doi.org/10.1371/journal.pone.0183725

Brandt, H. M., Vanderpool, R. C., Curry, S. J., Farris, P., Daniel-Ulloa, J., Seegmiller, L., Stradtman, L. R., Vu, T., Taylor, V., \& Zubizarreta, M. (2019). A multi-site case study of community-clinical linkages for promoting HPV vaccination. Human Vaccines \&amp; Immunotherapeutics, 15(7-8), 1599-1606. https://doi.org/10.1080/21645515.2019.1616501

Brotherton, J. (2019). Impact of HPV vaccination: Achievements and future challenges. Papillomavirus Research (amsterdam, Netherlands), 7, 138-140. https://doi.org/10.1016/j.pvr.2019.04.004

Brueggmann, D., Opper, N., Felix, J., Groneberg, D. A., Mishell, D. R., \& Jaque, J. M. (2016). Development of a cost-effective educational tool to promote acceptance of the HPV vaccination by hispanic mothers. Journal of Community Health, 41(3), 468-475. https://doi.org/10.1007/s10900-015-0116-z

Bryan, C. J., Bryan, A. O., \& Baker, J. C. (2020). Associations among state-level physical distancing measures and suicidal thoughts and behaviors among U.S. adults during the early COVID-19 pandemic. Suicide \&amp; Life-Threatening Behavior, 50(6), 1223-1229. https://doi.org/10.1111/sltb. 12653

Catalano, H. P., Knowlden, A. P., Birch, D. A., Leeper, J. D., Paschal, A. M., \& Usdan, S. L. (2017). Using the theory of planned behavior to predict HPV vaccination intentions of college men. Journal of American College Health: J of ACH, 65(3), 197-207. https://doi.org/10.1080/07448481.2016. 1269771

CDC. (2019). STD Facts-Human papillomavirus (HPV). https://www.cdc.gov/std/hpv/stdfact-hpv.htm

CDC. (2021). Understanding HPV Coverage. https://www.cdc.gov/hpv/partners/outreach-hcp/hpv-cover age.html

da Silva Oliveira, R. C., Albuquerque, U. P., da Silva, T. L. L., Júnior, W. S. F., Chaves, L. D. S., \& Araújo, E. D. L. (2017). Religiousness/spirituality do not necessarily matter: Effect on risk perception and adaptive strategies in the semi-arid region of NE Brazil. Global Ecology and Conservation, 11, 125-133. https://doi.org/10.1016/j.gecco.2017.05.004

Gordon, D., Waller, J., \& Marlow, L. A. (2011). Attitudes to HPV vaccination among mothers in the British Jewish community: Reasons for accepting or declining the vaccine. Vaccine, 29(43), 7350-7356. https://doi.org/10.1016/j.vaccine.2011.07.083

Hamilton, K., van Dongen, A., \& Hagger, M. S. (2020). An extended theory of planned behavior for parent-for-child health behaviors: A meta-analysis. Health Psychology: Official Journal of the Division of Health Psychology, American Psychological Association, 39(10), 863-878. https://doi.org/ 10.1037/hea0000940

Hamm, J., \& Yun, J. (2019). Influence of physical activity on the health-related quality of life of young adults with and without autism spectrum disorder. Disability and Rehabilitation, 41(7), 763-769. https://doi.org/10.1080/09638288.2017.1408708

Koenig, H. G., \& Büssing, A. (2010). The Duke University Religion Index (DUREL): A five-item measure for use in epidemological studies. Religions, 1(1), 78-85. https://doi.org/10.3390/rel1010078 
Kreuter, M. W., Fernandez, M. E., Brown, M., Cofta-Woerpel, L., Pfeiffer, D., Adams-Piphus, B., Krebill, H., Gonzalez, D. A., Campos, D. M., Kirklin, G. T., Betsworth, S., Casey, C., \& Luke, D. (2012). Increasing Information-seeking about human papillomavirus vaccination through community partnerships in African American and hispanic communities. Family \&amp; Community Health, 35(1), 15-30. https://doi.org/10.1097/FCH.0b013e3182385d13

Lahijani, A. Y., King, A. R., Gullatte, M. M., Hennink, M., \& Bednarczyk, R. A. (2021). HPV Vaccine promotion: The church as an agent of change. Social Science \&amp; Medicine, 268, 113375. https:// doi.org/10.1016/j.socscimed.2020.113375

Lee, H. O., \& Kim, S. (2015). Linking health information seeking to behavioral outcomes: Antecedents and outcomes of childhood vaccination information seeking in South Korea. Journal of Health Communication, 20(3), 285-296. https://doi.org/10.1080/10810730.2014.927035

Manika, D., Stout, P. A., Golden, L. L., \& Mackert, M. (2017). How does objective and subjective human papillomavirus knowledge affect information-seeking intentions and source preferences? Health Marketing Quarterly, 34(4), 247-267. https://doi.org/10.1080/07359683.2017.1375236

Marti, M., de Cola, M., MacDonald, N. E., Dumolard, L., \& Duclos, P. (2017). Assessments of global drivers of vaccine hesitancy in 2014-Looking beyond safety concerns. PLOS ONE, 12(3), e0172310. https://doi.org/10.1371/journal.pone.0172310

Meites, E., Kempe, A., \& Markowitz, L. E. (2016). Use of a 2-dose schedule for human papillomavirus vaccination-updated recommendations of the advisory committee on immunization practices. MMWR. Morbidity and Mortality Weekly Report, 65(49), 1405-1408. https://doi.org/10.15585/ mmwr.mm6549a5

Molina, Y., Silva, A., \& Rauscher, G. H. (2015). Racial/ethnic disparities in time to a breast cancer diagnosis: The mediating effects of health care facility factors. Medical Care, 53(10), 872-878. https:// doi.org/10.1097/MLR.0000000000000417

Mupandawana, E. T., \& Cross, R. (2016). Attitudes towards human papillomavirus vaccination among African parents in a city in the north of England: A qualitative study. Reproductive Health, 13(1), 97. https://doi.org/10.1186/s12978-016-0209-X

Näsman, A., Du, J., \& Dalianis, T. (2020). A global epidemic increase of an HPV-induced tonsil and tongue base cancer-potential benefit from a pan-gender use of HPV vaccine. Journal of Internal Medicine, 287(2), 134-152. https://doi.org/10.1111/joim.13010

Okunrounmu, E., Allen-Wilson, A., Davey, M., \& Davey, A. (2016). Black church leaders' attitudes about seeking mental health services: Role of religiosity and spirituality. International Journal of Religion \&amp; Spirituality in Society, 6(4), 45-55. https://doi.org/10.18848/2154-8633/cgp/v06i04/45-55

Olagoke, A. A., Olagoke, O. O., \& Hughes, A. M. (2020). Intention to vaccinate against the novel 2019 coronavirus disease: The role of health locus of control and religiosity. Journal of Religion and Health. https://doi.org/10.1007/s10943-020-01090-9

Paas, L. J., \& Morren, M. (2018). PLease do not answer if you are reading this: Respondent attention in online panels. Marketing Letters, 29(1), 13-21. https://doi.org/10.1007/s11002-018-9448-7

Padela, A. I., Malik, S., Vu, M., Quinn, M., \& Peek, M. (2018). Developing religiously-tailored health messages for behavioral change: Introducing the reframe, reprioritize, and reform ("3R") model. Social Science \&amp; Medicine, 204, 92-99. https://doi.org/10.1016/j.socscimed.2018.03.023

Perkins, R. B., Zisblatt, L., Legler, A., Trucks, E., Hanchate, A., \& Gorin, S. S. (2015). Effectiveness of a provider-focused intervention to improve HPV vaccination rates in boys and girls. Vaccine, 33(9), 1223-1229. https://doi.org/10.1016/j.vaccine.2014.11.021

Pratt, R., Njau, S. W., Ndagire, C., Chaisson, N., Toor, S., Ahmed, N., Mohamed, S., \& Dirks, J. (2019). "We are muslims and these diseases don't happen to us": a qualitative study of the views of young Somali men and women concerning HPV immunization. Vaccine, 37(15), 2043-2050. https://doi. org/10.1016/j.vaccine.2019.03.006

Shelton, R. C., Snavely, A. C., De Jesus, M., Othus, M. D., \& Allen, J. D. (2013). HPV Vaccine decisionmaking and acceptance: Does religion play a role? Journal of Religion and Health, 52(4), 11201130. https://doi.org/10.1007/s10943-011-9553-X

Soucy, J. N., \& Hadjistavropoulos, H. D. (2017). Treatment acceptability and preferences for managing severe health anxiety: Perceptions of internet-delivered cognitive behaviour therapy among primary care patients. Journal of Behavior Therapy and Experimental Psychiatry, 57, 14-24. https://doi.org/ 10.1016/j.jbtep.2017.02.002

Thomas, T., Blumling, A., \& Delaney, A. (2015). The influence of religiosity and spirituality on rural parents' health decision making and human papillomavirus vaccine choices. ANS. Advances in Nursing Science, 38(4), E1-E12. https://doi.org/10.1097/ANS.0000000000000094 
Touyz, S. J., \& Touyz, L. Z. (2013). The kiss of death: HPV rejected by religion. Current Oncology, 20(1), e52-e53. https://doi.org/10.3747/co.20.1186

Weinstein, J. E., Ananth, A., Brunner, J. P., Nelson, R. E., Bateman, M. E., Carter, J. M., Buell, J. F., \& Friedlander, P. L. (2016). Efficacy of a human papillomavirus vaccination educational platform in a diverse urban population. JAMA Otolaryngology Head \&amp; Neck Surgery, 142(6), 590-595. https://doi.org/10.1001/jamaoto.2016.0433

White, J. L. (2013). Logistic regression model effectiveness: Proportional chance criteria and proportional reduction in error. Journal of Contemporary Research in Education, 2(1), 3.

Williams, J., Fisher, M. P., Bayliss, E. A., Morris, M. A., \& O'Leary, S. T. (2020). Clergy attitudes toward vaccines and vaccine advocacy: A qualitative study. Human Vaccines \&amp; Immunotherapeutics, 16(11), 2800-2808. https://doi.org/10.1080/21645515.2020.1736451

Wong, L. P., Alias, H., Sam, I. C., \& Zimet, G. D. (2019). A Nationwide study comparing knowledge and beliefs about HPV among female students before and after HPV vaccination. Journal of Pediatric and Adolescent Gynecology, 32(2), 158-164. https://doi.org/10.1016/j.jpag.2018.10.010

Publisher's Note Springer Nature remains neutral with regard to jurisdictional claims in published maps and institutional affiliations. 